\title{
LA-UR-21-30479
}

Approved for public release; distribution is unlimited.

Title: $\quad$ DRiFT - Detector Response Function Toolkit Organic Scintillator and Gas Detector Capability Overview

Author(s): $\quad$ Andrews, Madison Theresa

Intended for: $\quad$ Distribute to interested external users of DRiFT

Issued: 
Disclaimer:

Los Alamos National Laboratory, an affirmative action/equal opportunity employer, is operated by Triad National Security, LLC for the National Nuclear Security Administration of U.S. Department of Energy under contract 89233218CNA000001. By approving this article, the publisher recognizes that the U.S. Government retains nonexclusive, royalty-free license to publish or reproduce the published form of this contribution, or to allow others to do so, for U.S. Government purposes. Los Alamos National Laboratory requests that the publisher identify this article as work performed under the auspices of the U.S. Department of Energy. Los Alamos National Laboratory strongly supports academic freedom and a researcher's right to publish; as an institution, however, the Laboratory does not endorse the viewpoint of a publication or guarantee its technical correctness. 


\section{DRiFT - Detector Response Function Toolkit Organic Scintillator and Gas Detector Capability Overview}

XCP-7: Radiation Transport Applications, XCP Division, Madison@lanl.gov

DRiFT Contributors include: Cameron Bates, Edward McKigney, Austin Mullen, Surafel Woldegiorgis, Michael Rising, Matthew Marcath, Avneet Sood 


\section{DRiFT - A Brief Overview}

- DRiFT post-processes MCNP output and simulates realistic nuclear instrumentation response.

- Modular - easily accommodates new instrumentation and physics models

- Easy to use - simple keyword input and one line execution, flexible ASCII write-out that can be post-processed.

- Capabilities: Organic Scintillators and Gas Detectors (primarily He-3).

- In this talk, features will not be described in detail, rather highlighted so you can get a feel for DRiFT capabilities.

- Split into four short sections: Overview, Scintillators, Gas Detectors, and Other Features.

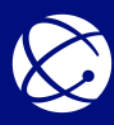




\section{Part I: DRiFT Overview}




\section{DRiFT Use and Input}

- Required: MCNP output (PTRAC)

- Distribution

- Executable for scintillators (new!)

- HPC build for gas detectors

- Input file

- Simple keywords for each module

- Output

- Text file output

- ROOT trees

\section{[global]}

modeltype=event

datasource=mcnp

ptrac type=bin

\#Name of the PTRAC file you want to process

datafile=omcnp_p

\#datafile is the file name of the monp ptrac output

det_cells=1

[SourceInformation]

call=SourceInformation

multi_src=yes

[Scintillation]

call=Scintillation

detector=EJ301

optical transport $=0.6$

pmt_type $=9821 \mathrm{~B}$

voltage $=1500$

divider option=B

[Digitizer]

call=Digitizer

voltage range $=2.0$

digitizer_samples $=256$

resolution $=16384$

ter res $=50$

DC offset $=0.1$

start_point $=0.1$

digitizer rate $=500$. e6

$\begin{array}{rrr}1.63259 & 71 & 0.133547 \\ 1.814 & 354 & 0.255438 \\ 3.29549 & 640 & 0.484216 \\ 1.66616 & 763 & 0.105647 \\ 0.879835 & 774 & 0.0920073 \\ 2.02652 & 1001 & 0.440321 \\ 2.76593 & 1016 & 0.606231\end{array}$

$\begin{array}{lr}\text { no } & 7.39562 \mathrm{e}-09 \\ \text { no } & 3.94077 \mathrm{e}-09 \\ \text { no } & 3.26886 \mathrm{e}-09 \\ \text { no } & 4.30608 \mathrm{e}-09 \\ \text { no } & 9.41205 \mathrm{e}-09 \\ \text { no } & 4.41343 \mathrm{e}-09 \\ \text { no } & 3.3331 \mathrm{e}-09\end{array}$




\section{Release - Creating a DRiFT Installer}

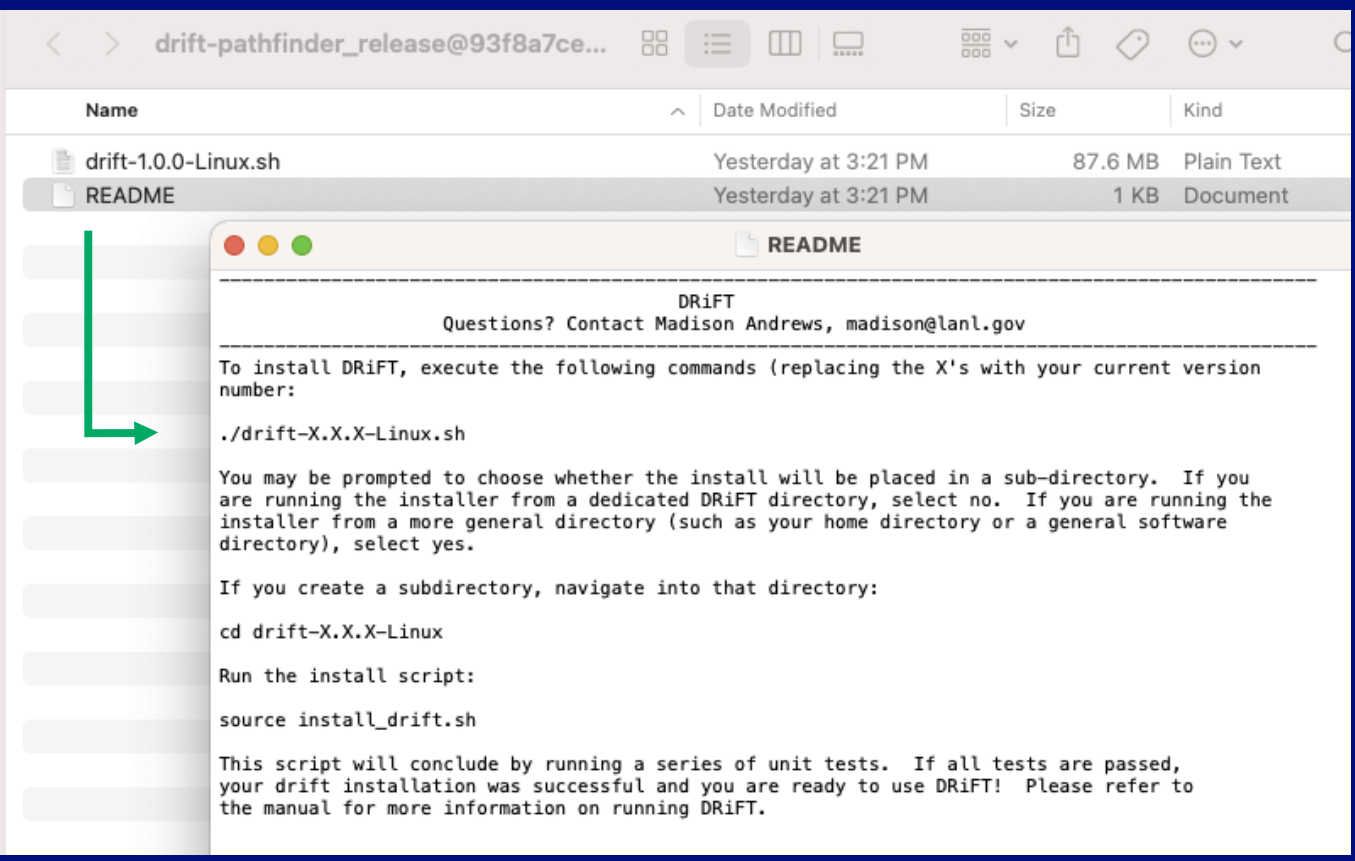

- A DRiFT executable installer is now available to approved requesters.

- It contains the installer (drift-1.0.0-Linux.sh) and a README file with simple installation instructions for the user.
- In order to generate a Linux executable:

- DRiFT dependencies on LANL's HPC cluster, ROOT, Garfield++ and MCNPTools builds were removed.

- CPACK was used to generate a STGZ self-extracting installer

- Remaining dependencies (HDF5 and GCC libraries) were statically linked

- The release contains 7 test suites / examples in addition to 3 unit tests.

- The unit tests are automatically executed at the end of the install process, and compare the output of various internal DRiFT functions against archived values. 


\section{Documentation - Manual}

DRiFT - RELEASE 1.0.0

ORGANIC SCINTILLATORS

DRIFT CONTRIBUTORS:

MAdison ANDREWS ${ }^{* 1}$, CAMERON BATES ${ }^{1}$, EdWARD MCKIGNEY ${ }^{1}$ AUSTIN MULLEN $^{1}$, SURAFEL WOLDEGIORGIS ${ }^{1}$, MiCHAEL RISING ${ }^{2}$, MATTHEW MARCATH ${ }^{1}$, AND AVNEET SOOD ${ }^{1}$

\footnotetext{
${ }^{1} X C P-7:$ Radiation Transport Application

${ }^{2} \mathrm{XCP}-3$ : Monte Carlo Codes

$X$-Computational Physics Division

Los Alamos National Laboratory

*madison@lanl.gov
}

- Detailed 65 page manual was created with TED funds to accompany the DRiFT executable.

- The manual contains 12 chapters split into 4 parts:

- DRiFT Overview

- Detector Physics - Scintillators

- Additional DRiFT Features

- Test Suites and Examples

- DRiFT executable and manual have been used by a friendly tester in nuclear safeguards in Q4 of FY21. 


\section{Generating Test Suites, Examples, and Unit Tests}

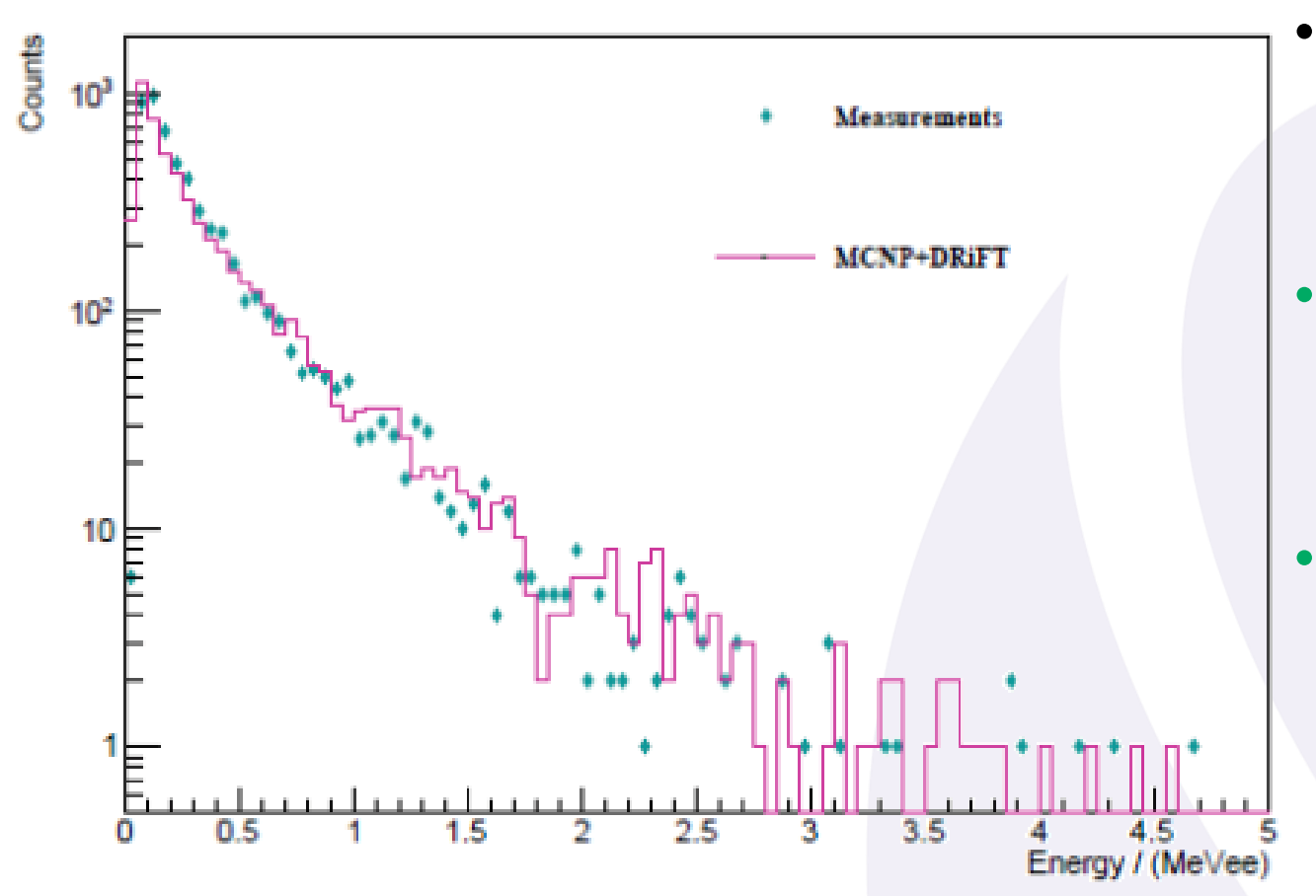

- 2 existing test suites were cleaned up, and documented.

- 5 new test suites / examples were added for the release.

Nuclear safeguards relevant examples include: correlated fission measurements, pile-up, cross talk, source activities, and comparisons of DRiFT with measurements (shown on left)

- Unit tests were developed to test code functionality upon installation.

- The 3 unit tests are automatically executed at the end of the installation process, and compare the output of various internal DRiFT functions against archived "truth" values generating using pre-determined inputs. 


\section{Part II: Organic Scintillators}




\section{Organic Scintillators in DRiFT}

- There are two large components to organic scintillator simulations in DRiFT: scintillator (and PMT) response, and digitizer effects (i.e. the conversion of electrons to a digitized signal).
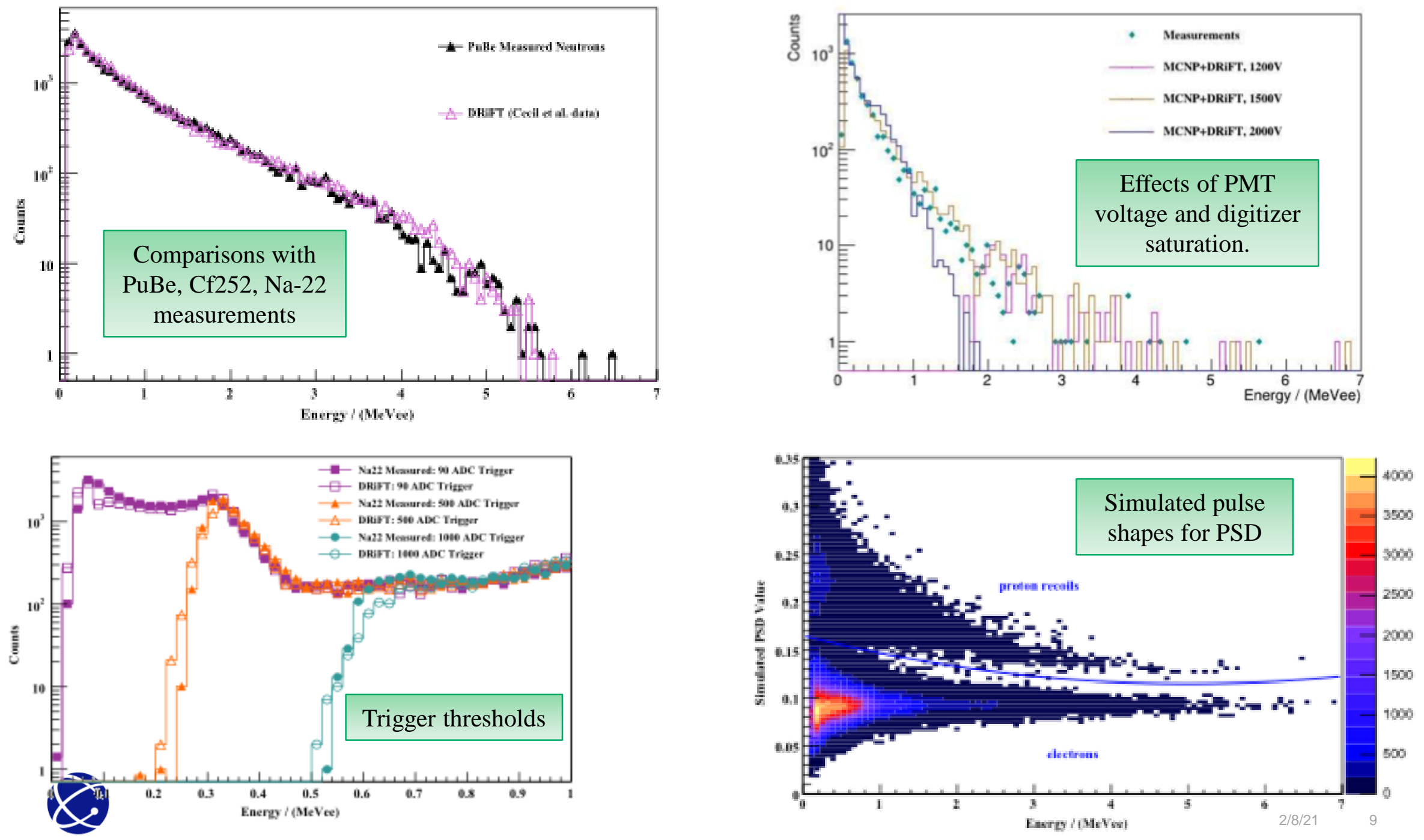


\section{Expansion of Scintillator and PMT Physics Options}

- Users can specify their own scintillator, PMT, and digitizer properties.

- Or, they can use models natively supported by DRiFT (17 scintillators, 13 PMTs, and 7 digitizer types).

- Additionally, the user can now add their own response without modifying source code.

- The release contains instructions and examples.

- Information required for users to "build their own" response is usually easily found on manufacturer's websites.

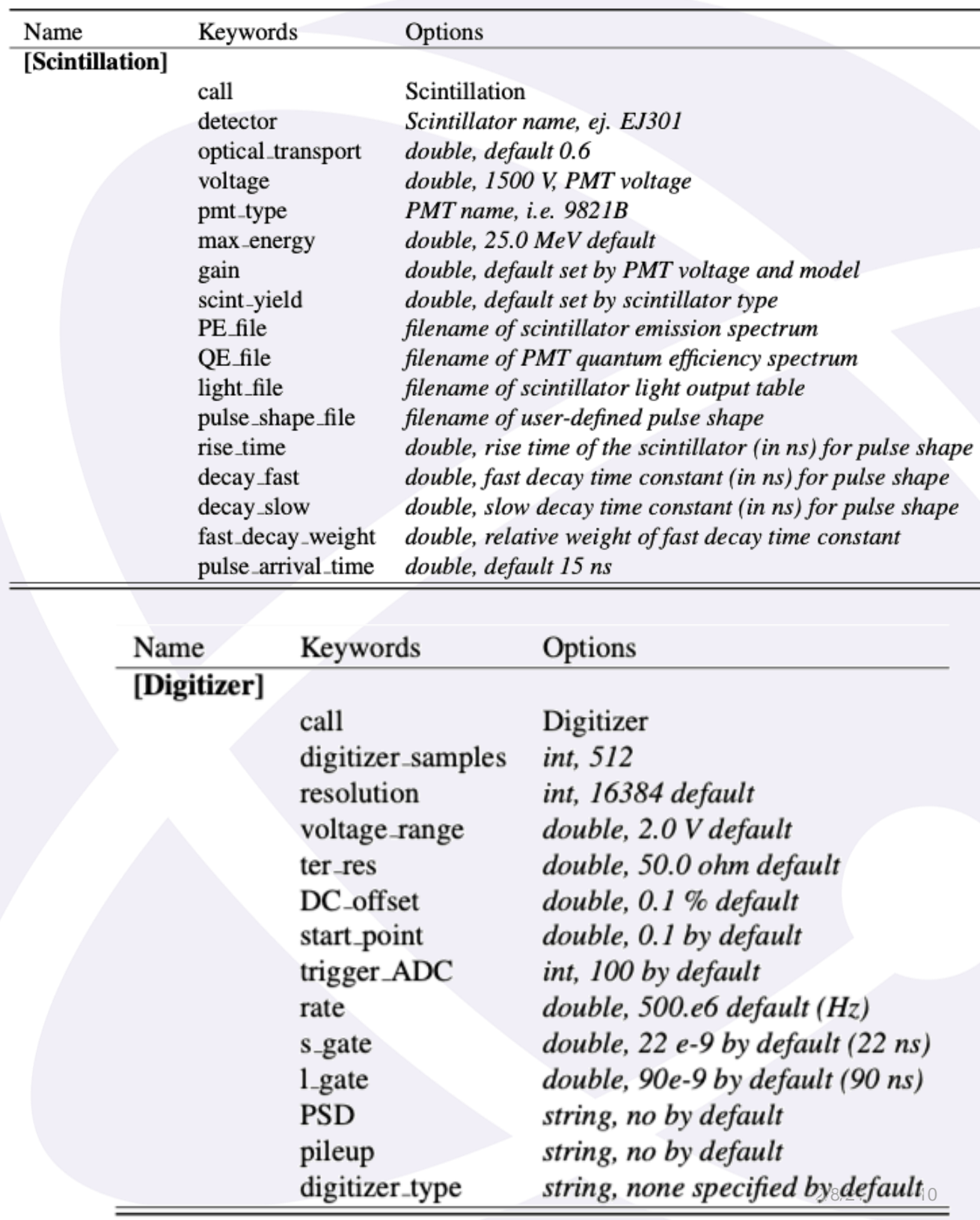




\section{Accommodating User Defined Pulse Shapes}

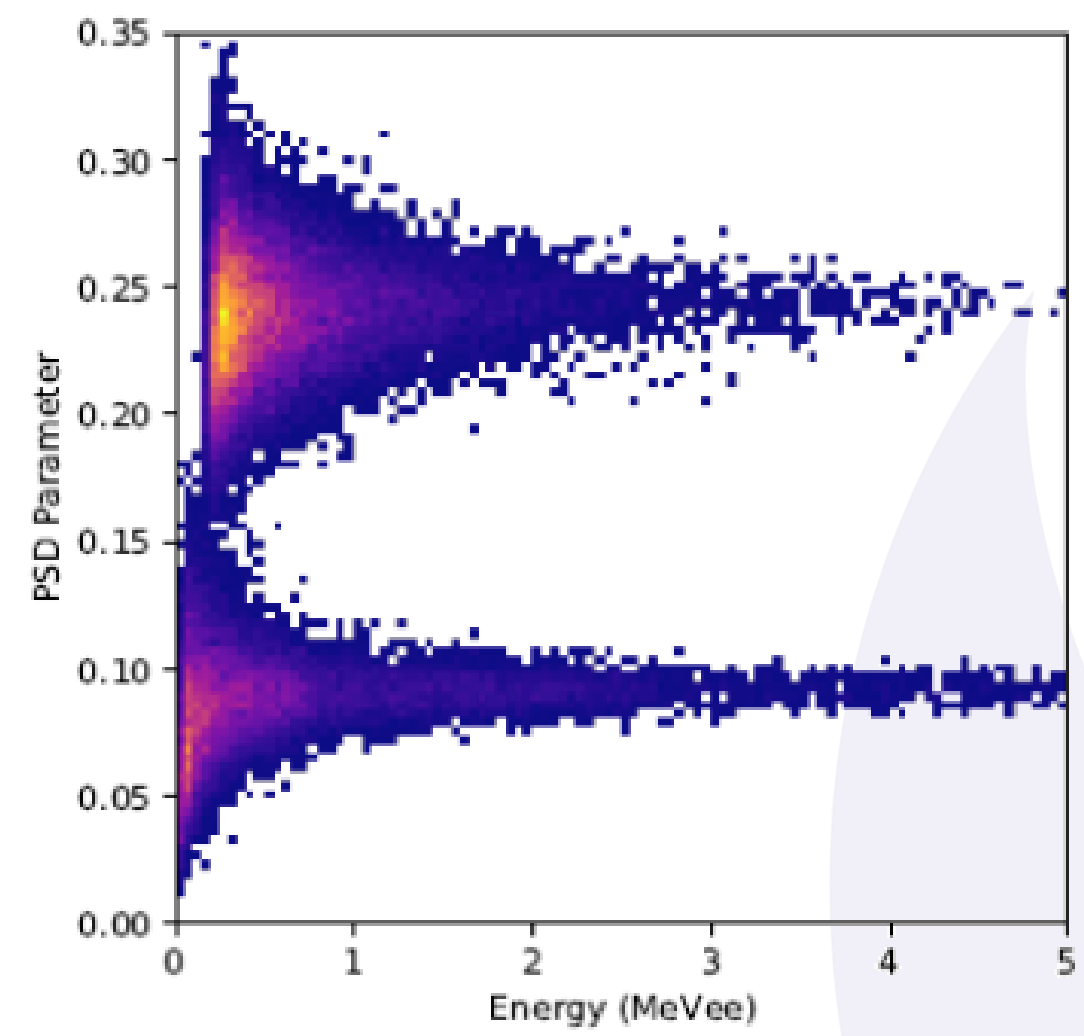

- One of the key useful and unique features of DRiFT is the ability to simulate digitizer electronic effects and pulses.

$-125$

- Simulated pulse shapes have a wide variety

100 of options from testing pulse shape

75 discrimination (PSD) analysis to generating testing data for machine learning algorithms.

$-25$

- Previously, only pulse shapes for EJ-301 scintillators were available.

- The code was expanded to accommodate user-defined pulses. An example of a PSD plot produced with this option is shown above.

- Users can define pulse shapes two ways: analytic equations or with an example measured pulse as drift input 


\section{Part III: Gas Detectors}




\section{Gas Detector - A new capability for DRiFT}

- Substantial progress has been made on helium-3 gas detector simulation capabilities and the gas detector proof of concept as been demonstrated.

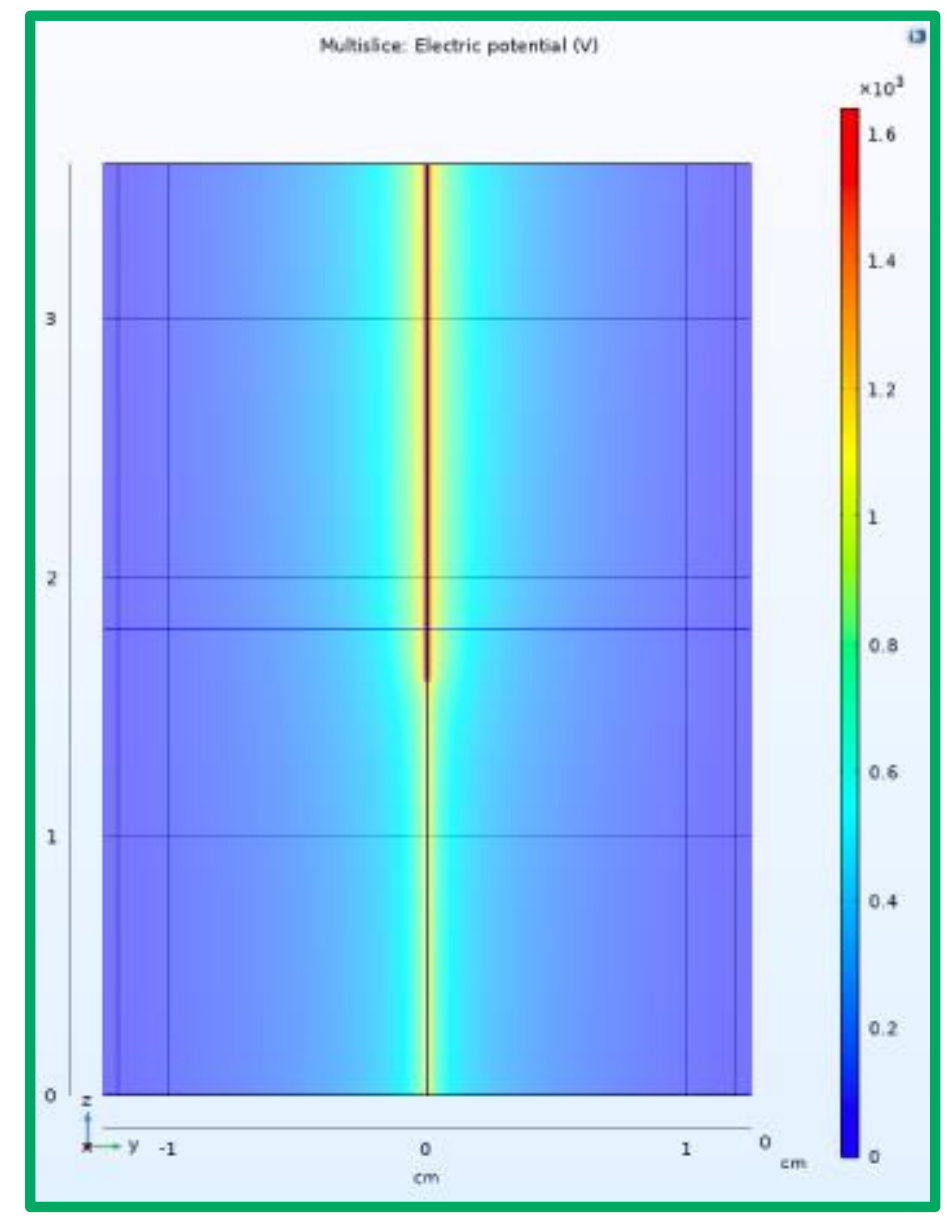

- Gas detector physics simulations necessitated output from many codes to create a DRiFT gas detector database (users do not need to interact with these external codes).

- COMSOL was used to model the tube electric fields in detail, these fields were imported into Garfield++ successfully.

- Incorporates field-tube (end-tube) effects.

- An interface to Magboltz computes gas properties, SRIM generates proton and triton stopping and range tables.

- Many combinations of tube pressures, sizes, quench gas, temperatures, and voltages to generate data tables that are read by DRiFT. 


\section{Gas Detectors}

- DRiFT account for decreases in detection efficiencies due to

- Operating at lower voltages

- The inactive tube area.

- Also added a pre-amplifier module compatible with both gas and semiconductor output, can give an estimate of pile-up, and photons misattributed as neutron events.

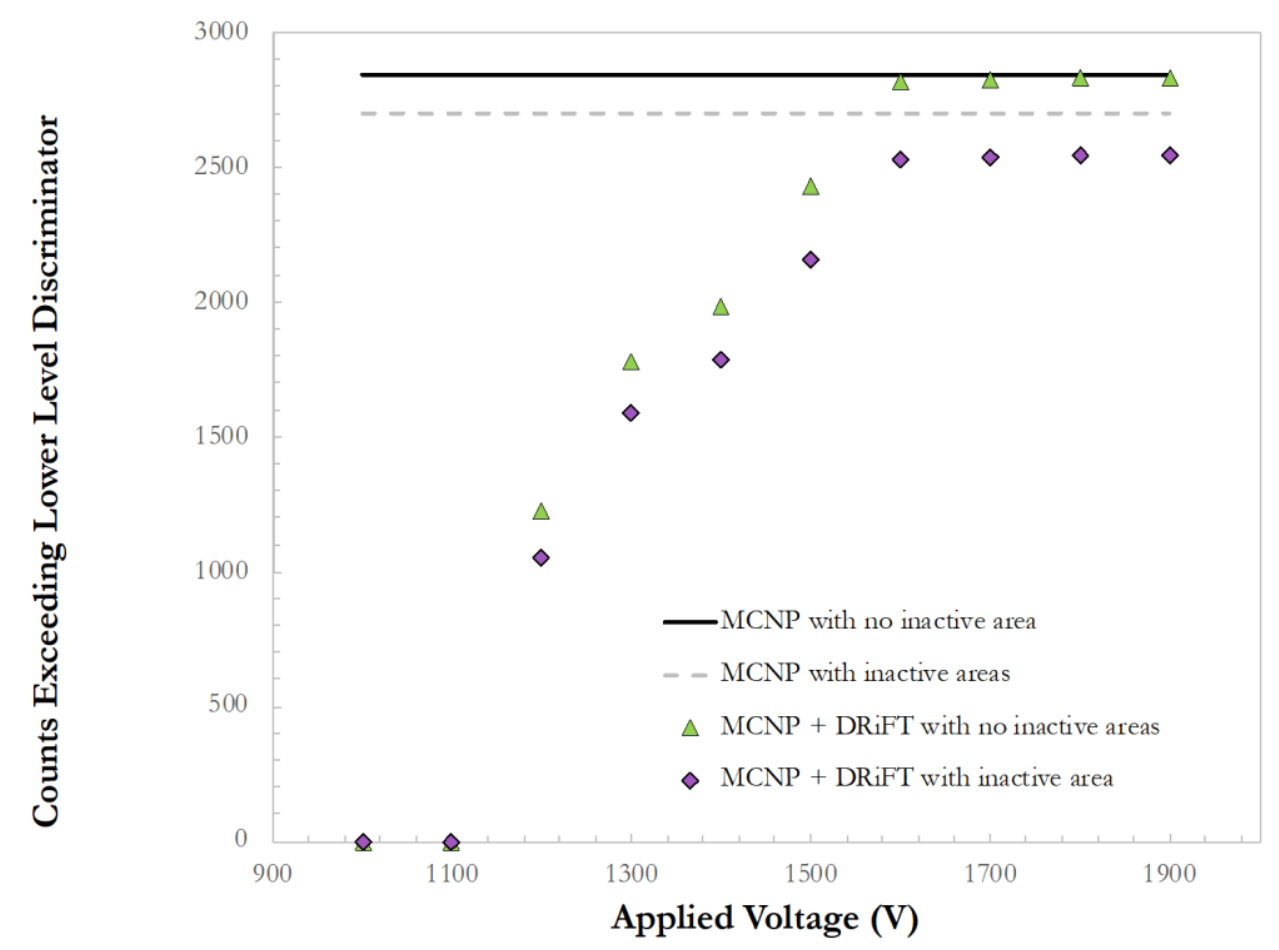

- Measurement sets to compare with DRiFT and MCNP would be appreciated if anyone has some relevant ones.

- Events can be discriminated based on energy deposited (like MCNP) or charge (more realistic). 


\section{Gas Options Implemented in DRiFT}

\begin{tabular}{|c|c|c|c|c|}
\hline Keyword & Description & Unit & Default & Options / Notes \\
\hline Gas1/Gas2 & Primary gas/quench gas & & Helium-3/CO $\mathrm{CO}_{2}$ & $\begin{array}{l}\mathrm{He}-3, \mathrm{BF} 3, \mathrm{CO}_{2} \text { whatever gases are supported in } \\
\text { Garfield++ (many), }\end{array}$ \\
\hline $\begin{array}{l}\text { Comp1/Comp } \\
2\end{array}$ & $\begin{array}{l}\text { Composition of primary/secondary } \\
\text { gas }\end{array}$ & Atom \% & 100 & $0-100$ \\
\hline Voltage & Voltage applied to the tube & V & 1600 & $1000-1900 \mathrm{~V}$ \\
\hline Pressure & Gas tube pressure & Atm & $10 \mathrm{~atm}$ & $1,2,4,10 \mathrm{~atm}$ \\
\hline Aval & $\begin{array}{l}\text { Model the electron transport in the } \\
\text { tube }\end{array}$ & & No & Yes, no \\
\hline Inactive area & Include end tube effects & & No & $\begin{array}{l}\text { Yes, no in Phase I a COMSOL + GARIFLED++ } \\
\text { generated file was required. }\end{array}$ \\
\hline $\begin{array}{l}\text { Inactive } \\
\text { bottom/ top }\end{array}$ & $\begin{array}{l}\text { Can describe the size of the inactive } \\
\text { areas on the top and bottom }\end{array}$ & $\mathrm{cm}$ & 0 & $\begin{array}{l}\text { With reserve funds we demonstrated that complex } \\
\text { COMSOL models were not necessary, significantly } \\
\text { increasing the flexibility of this feature. }\end{array}$ \\
\hline LLD_c & Lower level discriminator -charge & $\mathrm{C}$ & $0 \mathrm{C}$ & $\geq 0$, also added this to the pre-amplifier model \\
\hline LLD_e & Lower level discriminator - energy & $\mathrm{MeV}$ & $0 \mathrm{MeV}$ & $\geq 0$, also added this to the pre-amplifier model \\
\hline Temperature & Temperature of the tube & K & $293 \mathrm{~K}$ & Any \\
\hline srim & $\begin{array}{l}\text { Whether the tracks of the reaction } \\
\text { products are modelled }\end{array}$ & & Yes & Yes, No \\
\hline
\end{tabular}




\section{Part IV: Other Features in DRiFT}




\section{Information of source particles leading to detection events}

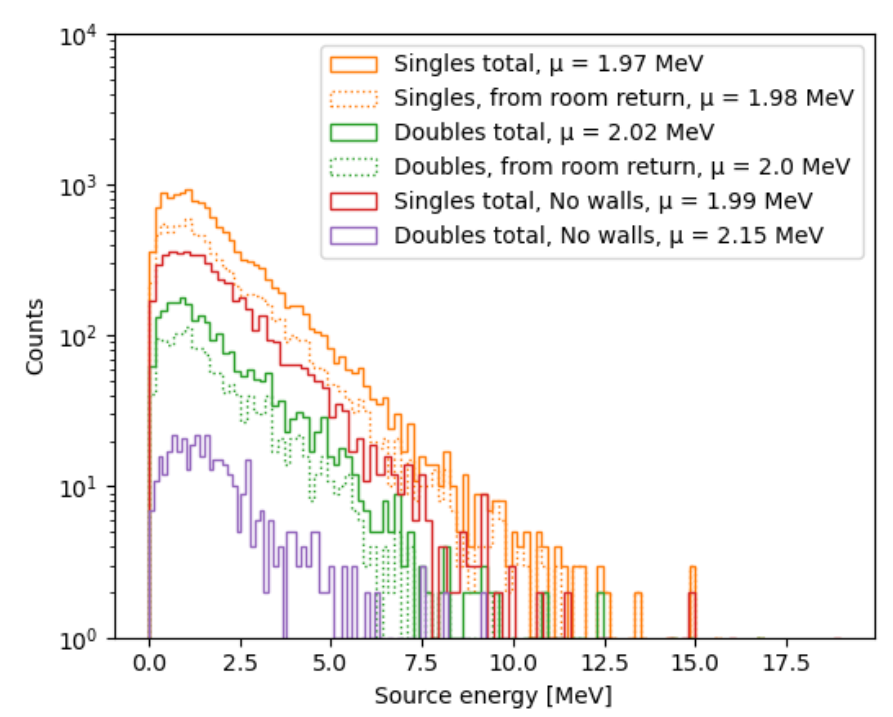

- Correlation between source energies/emission probabilities and capture moments in MC15, with and without room return contributions.

- DRiFT enables the tracking of particles from source point to final termination in detectors, allowing calculation of source energy and emission probabilities corresponding to each detection event.

- Using MCNPTools

- Potential input for unfolding and analysis codes

\section{Defining Source Activity in DRiFT}

- Users can define the source activity levels and DRiFT will automatically sort the PTRAC events in time accordingly.

- This allows an estimation of pile-up effects at a large number of detector settings.

- Options demonstrated include, pille-up, tube-end effects, collection time, energy thresholds and applied voltage.

- Currently DRiFT assumes a non-paralyzable deadtime in the pre-amplifier module. 


\section{Flexible Output}

Table 3.6: DRiFT Sections Keyword Options - WriteOutput

- The executable version can write out text files with columns corresponding to userspecified outputs and rows, each detector event.

- Can also output history of the particle (i.e. which cells in the MCNP file it interacted with).

\begin{tabular}{lll}
\hline Name & Keywords & Options \\
\hline [WriteOutput] & & WriteOutput \\
& call & integer less than 10 \\
& outputs & source_e (default), $\mathrm{MeV}$ \\
& & source_t seconds \\
& source_cell \\
& source_type \\
& count \\
& det_pulse \\
& det_cell \\
& corr_count \\
& PSD \\
\hline \hline
\end{tabular}

- Additionally, users can output digitizer waveforms for scintillator simulations to assess their analysis algorithms.

- The HPC version also have the option to output DRiFT results as root trees.
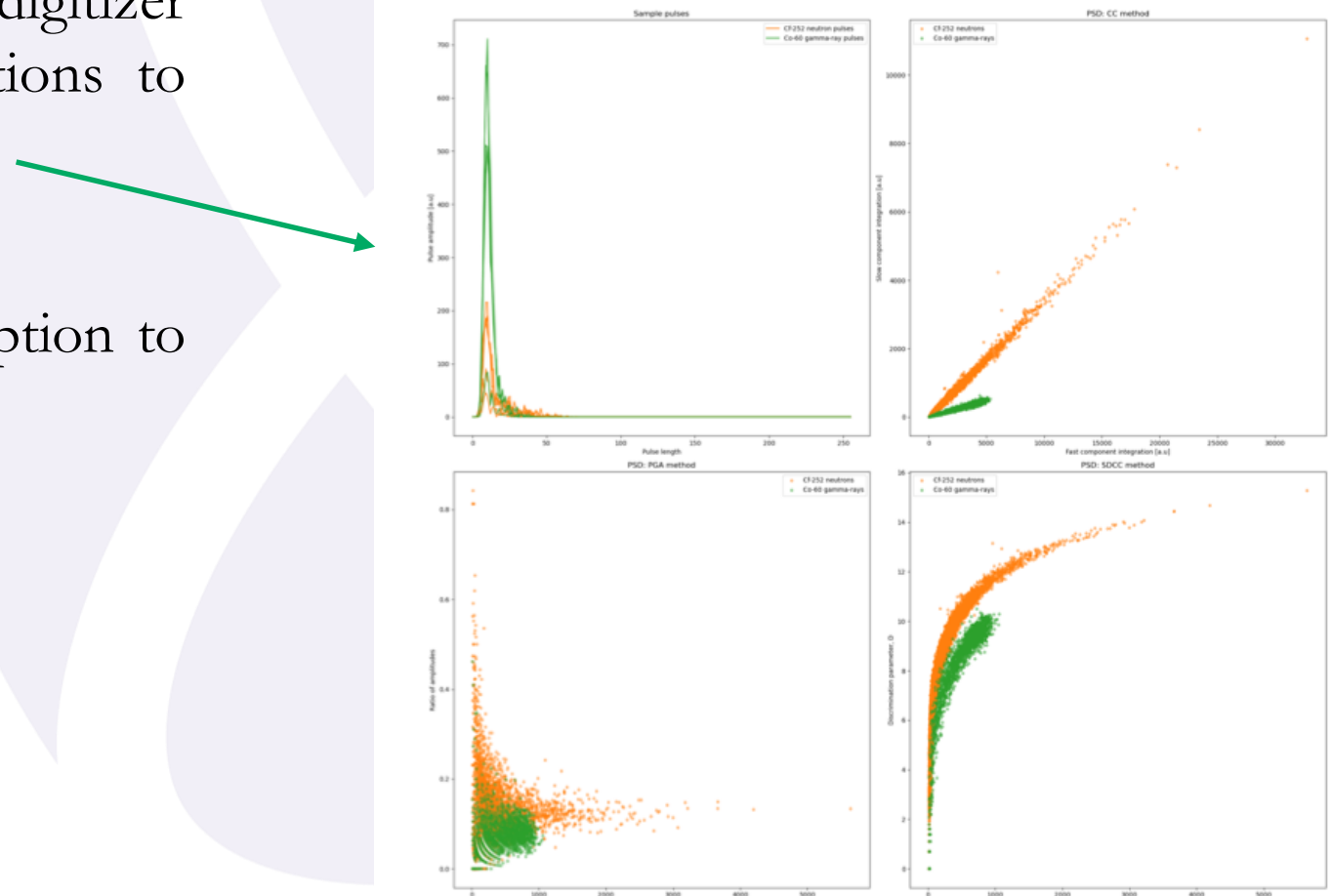


\section{Conclusions and Future Work}

- DRiFT simulates nuclear instrumentation in levels of detail not available in other codes.

- Allows users to assess tool performance and develop analysis algorithms (i.e. PSD).

- A DRiFT executable has been generated for Linux OS for organic scintillator capabilities.

- Gas detector proof of concept has been implemented.

- Future work:

- Streamlining of gas detector simulation process

- comparisons with He-3 measurements needed.

- Implementation of pulse shapes in gas detectors

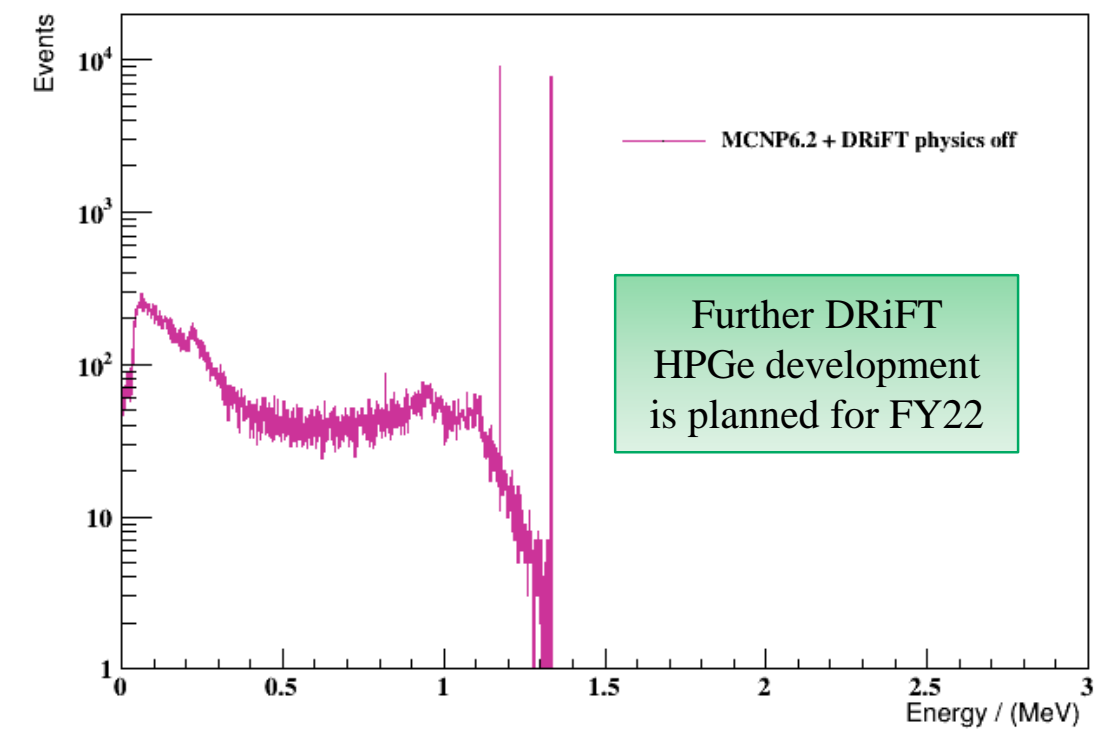

\section{Acknowledgements}

- LDRD MFR, TED Funds.

- We appreciate the contributions of measurements, information, feedback, testing, and MCNP decks from: J. Favorite, A. Madden, L. Misurek, M. Lombardi, K. Shults, M. James, T. Borgwardt, D. Broughton, S. Sarnoski, and M. Root. 